\title{
BMJ Open Prediction of exacerbation frequency of AECOPD based on next-generation sequencing and its relationship with imbalance of lung and gut microbiota: a protocol of a prospective cohort study
}

To cite: Deng L, Yan J, Xu H, et al. Prediction of exacerbation frequency of AECOPD based on next-generation sequencing and its relationship with imbalance of lung and gut microbiota: a protocol of a prospective cohort study. BMJ Open 2021;11:e047202. doi:10.1136/ bmjopen-2020-047202

- Prepublication history for this paper is available online. To view these files, please visit the journal online (http://dx.doi. org/10.1136/bmjopen-2020047202).

$\mathrm{LD}, \mathrm{JY}$ and $\mathrm{HX}$ contributed equally.

Received 21 November 2020 Accepted 28 July 2021

Check for updates

(c) Author(s) (or their employer(s)) 2021. Re-use permitted under CC BY-NC. No commercial re-use. See rights and permissions. Published by BMJ.

For numbered affiliations see end of article.

Correspondence to Professor Xiaoyin Chen; tchenxiaoyin@jnu.edu.cn

\section{ABSTRACT}

Introduction Patients with frequent acute exacerbation phenotype chronic obstructive pulmonary disease (AECOPD) have a higher hospitalisation rate than infrequent exacerbation, the disease progresses quickly and treatment is more difficult. At present, it is impossible to predict patients with COPD with frequent acute exacerbation phenotypes. The composition of the lower respiratory tract flora and the intestinal flora is closely related to AECOPD, but the specific association mechanism between them is not very clear. This study used metagenomic next-generation sequencing (mNGS) technology to explore the microbial characteristics of the intestinal tract and airways of patients with COPD, and analyse the correlation between the sequencing results and inflammatory factors, immune factors and nutritional factors.

Methods and analysis This will be a prospective cohort study. We intend to recruit 152 patients with stable COPD. In the baseline, we will detect the participants' induced sputum and faecal flora through mNGS, and changes in blood immune levels, and the patient's condition is evaluated. Every 2 months, we will check the number of acute exacerbation through the phone range. After 12 months, we will check again the changes in the blood immune level, evaluate the patient's condition and count the number of episodes.

Ethics and dissemination This study has been approved by the ethics committee of Guangdong Provincial Hospital of Traditional Chinese Medicine (approval number ZF2019219-03). The results of the study will be published in peerreviewed journals.

Trial registration number ClinicalTrials.gov Registry (ChiCTR2000032870).

\section{INTRODUCTION}

Chronic obstructive pulmonary disease (COPD) is one of the diseases with the highest morbidity and mortality in the world. Chronic and progressive dyspnoea is the most characteristic symptom of COPD. COPD exacerbation is defined by worsening symptoms

\section{Strengths and limitations of this study}

- This is a prospective study that uses the different phenotypes of chronic obstructive pulmonary disease (COPD) as an entry point. Metagenomic nextgeneration sequencing will be used to explore the microbial characteristics of the airways and gut of patients with COPD; the correlation between the sequencing results and inflammatory factors and nutritional factors will be conjointly analysed to predict the frequency of acute COPD attacks in the future.

- The influence and correlation of the intestinal flora on the lower respiratory tract flora will be discussed for the first time on acute exacerbation COPD (AECOPD)

- Phenotypical information of AECOPD includes data from CT scan, biosamples and clinical data from medical records.

- The outcome chosen, exacerbation frequency of AECOPD, is intuitive and objective.

Participants are recruited from a single centre.

(shortness of breath, cough) requiring steroids and antibiotics. ${ }^{1}$ Acute exacerbation of COPD (AECOPD) can be precipitated by several factors. The most common causes are respiratory tract infections. COPD exacerbation is a complex event usually associated with increased airway inflammation, increased mucus production and marked gas trapping. ${ }^{2}$ These changes contribute to increased dyspnoea that is the key symptom of exacerbation. Other symptoms include increased sputum purulence and volume, together with increased cough and wheeze. ${ }^{3} 4$ AECOPD can cause systemic inflammatory reactions in patients, with a significant decrease in respiratory function, skeletal muscle function decline and a significant decrease in mobility, which seriously affects the quality of life and has a poor prognosis. ${ }^{5}$ 
The COPD phenotype reflects the differences between individuals with $\mathrm{COPD},{ }^{6}$ and the COPD phenotype was associated with patients' symptoms, severity, treatment response, disease progression or mortality. The purpose of studying the COPD phenotype is to divide it into different subgroups to guide treatment so as to improve the prognosis of the disease, reduce the burden of the disease and improve the quality of life of patients. Clinically, patients with frequent acute exacerbation have a higher hospitalisation rate than those with infrequent exacerbation, and their disease progresses quickly, making treatment more difficult.

The susceptibility and frequent occurrence of COPD are based on multiple factors. Sometimes antibiotics are needed to fight infections that make COPD worse. One common side effect of antibiotic treatment for AECOPD is collateral damage to the gut microbiota composition, resulting in a possible overgrowth of opportunistic pathogenic bacteria. Pathogenic bacteria can disrupt the mucosal barrier by secretion of toxins, transfer of effector proteins into host cells, invasion of epithelial cells, translocation across the epithelial monolayer or by disruption of intercellular junctions, which further increase local and systemic inflammation. It has been demonstrated that intestinal dysfunction is present in COPD, worsens with increasing disease severity and is associated with reduced quality of life. ${ }^{7}$ In addition, individuals with COPD have functional and structural alterations in their intestinal mucosa, and patients with COPD typically have increased intestinal permeability. ${ }^{8-10}$ Increasing evidence has indicated the intimate relationship between the gastrointestinal tract and respiratory tract. ${ }^{11}$ The gut microbiota interacts with the host immune system in ways that influence disease development. ${ }^{10}$ On the other hand, sputum, alveolar lavage fluid, etc also contain specific components of respiratory tract microorganisms, which can cause the immune system to produce different immune responses. Through the evaluation of the airway microorganisms and their immune function, it can provide new ideas for more accurate targeting of antibiotics and enhancing the efficacy of immune responses. However, few studies have evaluated these important pathophysiological components of COPD to predict exacerbation risk. There are also few studies to observe the relationship between the changes in the intestinal flora of AECOPD and the changes in the lung flora.

The gut microbiota influences the AECOPD, but the mechanism between the gut microbiota and the development of COPD is not yet clear. Whether the changes in the gut microbiota and the changes in the pulmonary flora are related has not yet been studied. We hypothesise that the frequent exacerbation phenotype of COPD may be driven by a variety of gut microbiota and metabolites, and that the gut microbiota affects the changes in lung flora and is related to specific biomarkers. In this study, we will use metagenomic sequencing to clarify the relationship between gut microbiota, intestinal fungi and lung flora, and use metabonomics to analyse qualitatively
Box 1 Study inclusion and exclusion criteria

\section{Eligibility criteria}

- Patients diagnosed with chronic obstructive pulmonary disease (COPD). The diagnostic criteria for COPD are based on the 2020 Global Initiative for the Diagnosis, Treatment and Prevention of Chronic Obstructive Pulmonary Disease (GOLD Guidelines 2020). ${ }^{12}$ The diagnostic criteria for acute exacerbation of COPD (AECOPD) refer to the Chinese Expert Consensus on the Diagnosis and Treatment of AECOPD. ${ }^{40}$ Incompletely reversible airflow limitation is a prerequisite for COPD diagnosis. After inhaling bronchodilators,

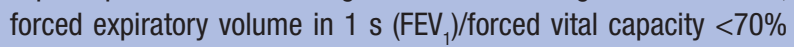
and $\mathrm{FEV}_{1}<80 \%$ predicted value can be determined as incompletely reversible airflow limitation.

- No antibiotics have been used in the past month.

- The patient has informed consent and signed an informed consent form, and can accept observation and clinical data collection.

\section{Exclusion criteria}

- Suffer from chronic wasting diseases such as tumour, tuberculosis and bronchiectasis at the same time.

- Combined with ulcerative colitis, Crohn's disease or other intestinal diseases.

- Those who have taken systemic glucocorticoids or immunosuppressants within 3 months.

- Those who have taken probiotics, prebiotics, and other microbial preparations regularly or vitamin supplements in the past.

- Poor compliance and unable to cooperate with clinical observation and specimen collection.

and quantitatively the metabolites of intestinal microbes in the blood. This study will establish a specific marker for exacerbation frequency of AECOPD, combine metagenomics data, and use systems biology methods to evaluate multiple specific intestinal flora representing multiple pathobiological mechanisms of COPD, especially for the evaluation of the impact of the gut and respiratory microbiome on prediction of exacerbation frequency of COPD.

\section{METHODS AND ANALYSIS \\ Study design}

The programme follows the guidelines of '2020 Global Initiative for the Diagnosis, Treatment and Prevention of Chronic Obstructive Pulmonary Disease (GOLD)'. ${ }^{12}$ The diagnostic criteria for COPD are based on the GOLD Guidelines 2020. Participants will participate in a baseline inpatient visit and follow-up every 2 months thereafter for a period of 12 months. The influence of host genetics, airway inflammation and respiratory microbiome on predicting the onset of COPD will be examined. The study inclusion and exclusion criteria are shown in box 1 .

At the end of the clinical trial, the group with common moderate-to-severe exacerbation and that with uncommon (less than two episodes of moderate-to-severe exacerbation) episodes will be compared. The primary outcomes are total number of exacerbation, acute exacerbation and time to first exacerbation, and the secondary 
outcomes are changes in lung function and symptom scores.

In this study, acute exacerbation is identified as patients diagnosed with COPD who have the following symptoms within a period of time: cough; sputum production; shortness of breath and/or wheezing worsened, increased sputum volume, purulent or mucopurulent, with or without fever. The total number of exacerbation is defined as the number of acute exacerbation in the next 12 months after being included in the trial. Time to first exacerbation is defined as the time from the first day when the patient is enrolled in the trial until the patient's first appearance of acute exacerbation. The definition of moderate exacerbation and severe exacerbation is based on the 'Chinese Experts' Chronic Consensus on Anti-infection Treatment in the Acute Exacerbation of Obstructive Pulmonary Disease' according to the patient's need for additional treatment measures to determine whether it is moderate exacerbation or severe exacerbation. COPD exacerbation is defined as acute worsening of respiratory symptoms that result in additional therapy. These events are classified as mild (treated with shortacting bronchodilators (SABDs) only), moderate (treated with SABDs plus antibiotics and/or oral corticosteroids) or severe (patient requires hospitalisation or visits the emergency room). Severe exacerbation may also be associated with acute respiratory failure. When the patient had acute exacerbation, the participant was asked to contact the research assistant and would be referred to the respiratory doctor in our hospital to assess the severity of the patient's onset. All patients will study the process as shown in figure 1 .

\section{Study settings}

This study will be completed in Guangdong Provincial Hospital of Traditional Chinese Medicine.

\section{Ethical approval}

This study was approved by the ethics committee of Guangdong Provincial Hospital of Traditional Chinese Medicine (approval number ZF2019-219-03). Patients who met the inclusion criteria will be enrolled after obtaining signed informed consent.

\section{Patient and public involvement}

Patients and the public were not and will not be involved in the design, conduct and reporting of the research.

\section{Clinical assessments}

\section{Baseline visit}

1. General data collection: name, gender, age, height, weight, occupation, place of residence, contact information, education level, marital status, medical history, family history, etc.

2. COPD-related data collection: spirometry, COPD staging, number of acute exacerbation before admission in the past 12 months, whether they have been exposed to risk factors, smoking history, current medication,

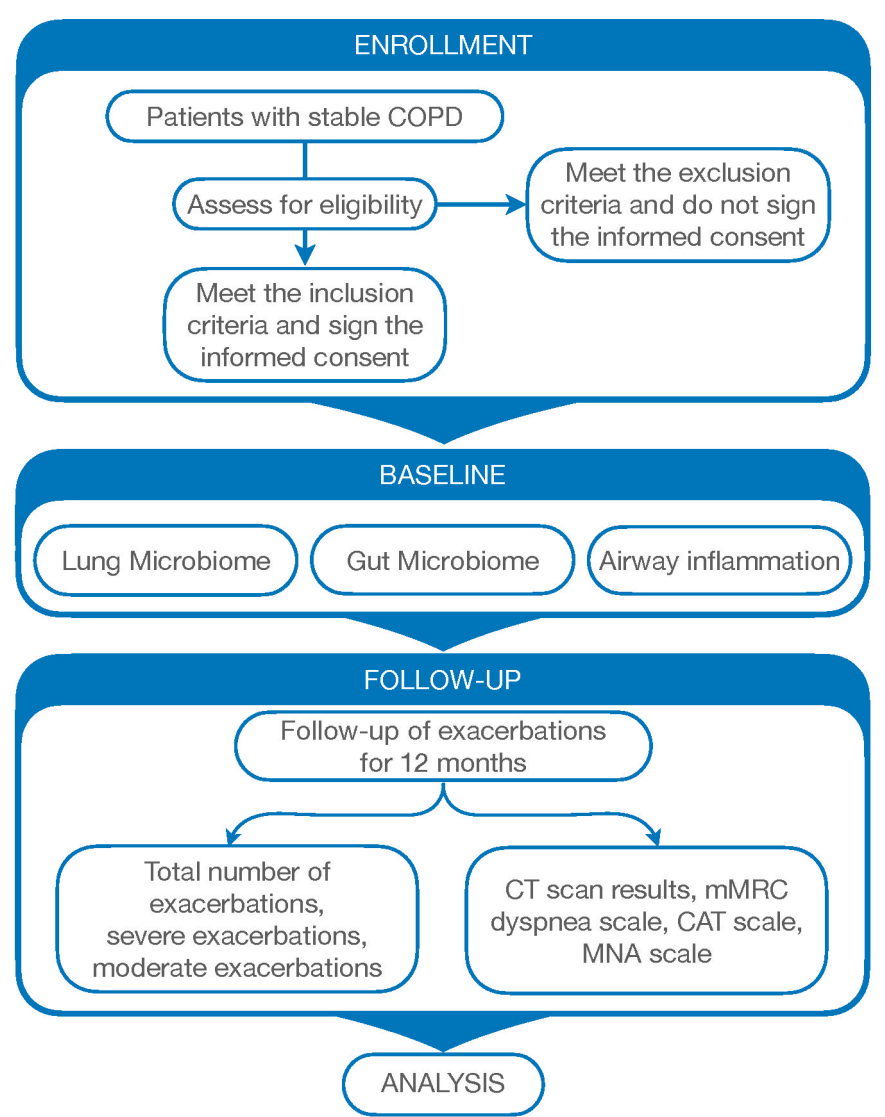

Figure 1 Flow chart of the study. CAT, COPD Assessment Test; COPD, chronic obstructive pulmonary disease; mMRC, modified Medical Research Council; MNA, Micro-Nutrition Assessment.

oxygen therapy, whether their disease is combined with other diseases, lung function, etc.

3. Imaging data collection: including chest radiograph, high-resolution CT scan, etc.

4. Information collection of scales: modified Medical Research Council (mMRC) dyspnoea scale, COPD Assessment Test (CAT) scale, Micro-Nutrition Assessment.

\section{Follow-up and acute exacerbation}

Patients with COPD have increased airway mucus secretion and increased airflow obstruction due to airway infection or non-infectious factors, which are manifested as increased dyspnoea, increased sputum volume and purulent sputum, often accompanied by increased cough and wheezing. When the degree of aggravation of the above symptoms exceeds the daily variation and reaches the level that requires intensive treatment or even medical treatment, it is AECOPD. After the patient was discharged from the hospital for the first time, the research assistant will be responsible for each patient's initial visit and telephone follow-up every 2 months. When the patient had acute exacerbation, the participant was asked to contact the research assistant and would be referred to the respiratory doctor in our hospital to assess the severity of the patient's onset. 
Table 1 Data collection schedule

\begin{tabular}{|c|c|c|c|c|c|c|c|}
\hline Stage visit and follow-up timeline & Visit 1 & $\begin{array}{l}\text { Phone } \\
\text { interview } 1 \\
\text { (2 months) }\end{array}$ & $\begin{array}{l}\text { Phone } \\
\text { interview } 2 \\
\text { (4 months) }\end{array}$ & $\begin{array}{l}\text { Phone } \\
\text { interview } 3 \\
\text { (6 months) }\end{array}$ & $\begin{array}{l}\text { Phone } \\
\text { interview } 4 \\
\text { (8 months) }\end{array}$ & $\begin{array}{l}\text { Phone } \\
\text { interview } 5 \\
\text { (10 months) }\end{array}$ & $\begin{array}{l}\text { Visit } 2 \text { (12 } \\
\text { months) }\end{array}$ \\
\hline $\begin{array}{l}\text { Collection of basic information } \\
\text { informed consent }\end{array}$ & $\sqrt{ }$ & & & & & & \\
\hline Inclusion and exclusion criteria & $\sqrt{ }$ & & & & & & \\
\hline COPD staging & $\sqrt{ }$ & & & & & & $\sqrt{ }$ \\
\hline Smoking history & $\sqrt{ }$ & & & & & & $\sqrt{ }$ \\
\hline Current medication & $\sqrt{ }$ & $\sqrt{ }$ & $\sqrt{ }$ & $\sqrt{ }$ & $\sqrt{ }$ & $\sqrt{ }$ & $\sqrt{ }$ \\
\hline $\begin{array}{l}\text { Chest radiograph and high-resolution } \\
\text { CT scan }\end{array}$ & $\sqrt{ }$ & & & & & & $\sqrt{ }$ \\
\hline $\begin{array}{l}\text { Information collection of scales } \\
\text { (mMRC, CAT, MNA) }\end{array}$ & $\sqrt{ }$ & & & & & & $\sqrt{ }$ \\
\hline Exacerbation & & $\sqrt{ }$ & $\sqrt{ }$ & $\sqrt{ }$ & $\sqrt{ }$ & $\sqrt{ }$ & $\sqrt{ }$ \\
\hline $\begin{array}{l}\text { mNGS for induced sputum, } \\
\text { pharyngeal swab and stool }\end{array}$ & $\sqrt{ }$ & & & & & & \\
\hline \multicolumn{8}{|l|}{ Blood sampling } \\
\hline Inflammatory cell counting & $\sqrt{ }$ & & & & & & $\sqrt{ }$ \\
\hline Blood routine examination & $\sqrt{ }$ & & & & & & $\sqrt{ }$ \\
\hline Arterial blood gas analysis & $\sqrt{ }$ & & & & & & $\sqrt{ }$ \\
\hline T lymphocyte subsets & $\sqrt{ }$ & & & & & & $\sqrt{ }$ \\
\hline $\lg A, \lg G, \lg M, C 3, C 4$ & $\sqrt{ }$ & & & & & & $\sqrt{ }$ \\
\hline
\end{tabular}

CAT, COPD Assessment Test; COPD, chronic obstructive pulmonary disease; mMRC, modified Medical Research Council; MNA, MicroNutrition Assessment; mNGS, metagenomic next-generation sequencing.

The data collection schedule is shown in table 1 . The definition of moderate exacerbation and severe exacerbation is based on the 'Chinese Experts' Chronic Consensus on Anti-infection Treatment in the Acute Exacerbation of Obstructive Pulmonary Disease' according to the patient's need for additional treatment measures to determine whether it is moderate exacerbation or severe exacerbation. For moderate exacerbation, use SABDs and antibiotics and/or oral hormones. It is severe exacerbation when hospitalisation or emergency treatment is required, and it may be accompanied by acute respiratory failure.

\section{Specimen collection}

1. Induced sputum: after being assessed by the attending physician, it will be collected by a specialist in the Department of Respiratory Medicine of Guangdong Provincial Hospital of Traditional Chinese Medicine. The collection and preparation of induced sputum refer to the improved method. ${ }^{13}{ }^{14}$ Before induction, the patient would rinse his mouth thoroughly with saline, swallow an appropriate amount of saline, and clear the mouth and nose secretions. Sputum induction was preceded by premedication with $400 \mu \mathrm{g}$ of inhaled salbutamol. After post-bronchodilator spirometry, the patient would inhale sterile hypertonic saline $(\mathrm{NaCl})$ at increasing concentrations $(3 \%, 4 \%$ and $5 \%$ solutions, $10 \mathrm{~min}$ per inhalation) via an ultrasonic nebuliser (ULTRA-NEB TM 2000, DeVilbiss, USA). After each inhalation, spirometry was performed to detect a potential forced expiratory volume in $1 \mathrm{~s}\left(\mathrm{FEV}_{1}\right)$ decrease. The procedure was stopped when a significant $(\geq 20 \%)$ $\mathrm{FEV}_{1}$ decline as compared with post-bronchodilator baseline value was noted. After the nebulisation, a sterile container was used to collect the induced sputum and quickly store it in a $-80^{\circ} \mathrm{C}$ refrigerator within 1 hour. The net weight of sputum should be greater than $0.1 \mathrm{~g}$. A part of the sputum should be used to complete the sputum smear microscopy. Giemsa staining method was used to observe the number of squamous epithelial cells in each low-power field. If the ratio of squamous epithelial cells is more than $20 \%$, the specimen is deemed unqualified. If qualified, cell classification and counting is possible; another part of the sputum is sent to Beijing Boao Inspection Company, and the sample storage container must indicate the sampling date and sample number, and it must be consistent with the information on the test application form. Specimens should be collected on the day of admission or the next day.

2. Pharyngeal swab: the specimen was collected by the Department of Respiratory Medicine of Guangdong Provincial Hospital of Traditional Chinese Medicine, in a special container for throat swab. Before the collection, the patient can rinse their mouth with normal saline. Gently scrape the patient's posterior pharynx, 
tonsil crypts, etc, repeatedly wipe three to five times. A total of five tubes is needed to collect mucosal cells. Avoid contact with tongue, oral mucus and saliva when taking it out. After the specimens are collected, they are stored in a refrigerator at $-20^{\circ} \mathrm{C}$ and delivered together with the induced sputum. Specimens should be collected on the day of admission.

3. Stool: the subject keeps two stool specimens by himself. After defecation, the patient is instructed to avoid picking the part that is in contact with the bottom and edge of the bedpan, placing it in the stool collection tube, and flicking the stool to the bottom of the stool collection tube. There should be three tubes. In addition, a common stool collection tube was used to collect one stool, stored in the refrigerator at $-4^{\circ} \mathrm{C}$, and sent to the laboratory of our hospital. Three tubes of stool collection were stored in the refrigerator at $-20^{\circ} \mathrm{C}$, and delivered together with the induced sputum and throat swabs. Specimens should be collected within 1-3 days after admission as far as possible. The patient should eat more foods with high water content and drink more water before defecation to help the smooth discharge of stool.

4. Blood: an appropriate amount of blood samples (arterial blood +venous blood) should be sent to the laboratory of our hospital immediately after being collected by the Department of Respiratory Medicine of Guangdong Provincial Hospital of Traditional Chinese Medicine. If the sample cannot be sent within 1-2 hours, the sample should be placed in the refrigerator for storage time but must not exceed 24 hours.

\section{Metagenomic sequencing \\ DNA extraction}

Induced sputum, pharyngeal swab and stool samples were collected from patients according to standard procedures. The process of metagenomic sequencing consisted of sample processing, nucleic acid extraction, library generation and bioinformatic pipeline analysis as described previously. ${ }^{1516}$ Total bacterial DNA was isolated and purified using QIAamp DNA Stool Mini Kit (Qiagen, Valencia, California, USA).

\section{Library construction and sequencing}

A sequencing library was constructed following the BGISEQ-500 instruction and using the standard protocol with minor modification. In brief, the genomic DNA was fragmented and DNA fragments between $100 \mathrm{bp}$ and $\sim 300$ bp were selected. The selected DNA fragments were repaired and modified. A dTTP tailed adapter sequence was ligated to both ends of the DNA fragments, and the fragments were further amplified and subjected to single-strand circularisation. Two types of sequencing strategies, paired-end (PE) and single-end (SE), were followed using the BGISEQ-500 platform with read length of $50 \mathrm{bp}$ and $100 \mathrm{bp}$, respectively (insert size $\sim 250 \mathrm{bp}$ ).
Metagenomic sequences de novo assembly

High-quality reads from each DNA sample were selected for de novo assembly of each sample. High-quality reads of PE50 and SE100 from each sample were assembled into longer contigs using the IDBA-UD (V.1.1.3) by iterated Kmer. Contigs constructed at each round of iteration were used as long reads for the next iteration.

\section{Establishment of a gene catalogue of microbiome}

The assembled sequences of each of the samples were filtered with a length exceeding 500 bases. These contigs were used for prediction of open reading frames (ORFs) using Prodigal (V.2.6.1) with the procedure 'meta'. In order to bin orthologues and avoid inflation of possible sequencing errors, shared ORFs were grouped using CD-HIT with a criterion of $95 \%$ identity $>90 \%$ of the shorter ORF length with default parameter except ' $-\mathrm{G} 0$ -n $8-\mathrm{aS} 0.9-\mathrm{c} 0.95-\mathrm{d} 0-\mathrm{g} 1$ '. The longest ORF in each group was selected to represent the group, and other members of the group were considered redundant.

\section{T lymphocyte subset detection}

Related methods are described in our previous article. ${ }^{17}$ Briefly, peripheral blood mononuclear cells (PBMCs) are separated and then stained with antibodies. T lymphocyte subsets were detected by flow cytometry.

\section{CAT score}

The evaluation of CAT score and severity of illness is as follows: CAT score $\leq 10$ : mild; $10<$ CAT score $\leq 20$ : moderate; $20<\mathrm{CAT}$ score $\leq 30$ : severe; CAT score $>30$ : very serious. $^{18}$

\section{mMRC dyspnoea score}

The evaluation of mMRC dyspnoea score is as follows: grade 1, not troubled with breathlessness except with strenuous exercise; grade 2, troubled by shortness of breath when hurrying on the level or walking up a slight hill; grade 3, walks slower than people of the same age on the level because of breathlessness or has to stop for breath when walking at own pace on the level; grade 4, stops for breath after walking about 100 yards or after a few minutes on the level; grade 5 , too breathless to leave the house or breathless when dressing or undressing. ${ }^{19}$

\section{Sample size calculation}

Sample size was calculated based on the primary outcome of exacerbation frequency. According to previous studies $^{2021}$ and our clinical observations, the probability of acute exacerbation of two or more times is $35 \%$, and the probability of acute exacerbation or no recurrence is $65 \%$.

By using the PASS software, we set the $\alpha=0.05$ and the power $1-\beta=0.9$. The ratio of cases among each group is $1: 2$, the number of cases calculated is 129 in total. We suppose a $30 \%$ of lost in the follow-up of the study, thus, the ultimately needed case is 168 . 


\section{Safety evaluation}

Before the induced sputum examination, prepare rescue equipment and medicines, and fully explain the whole process and precautions of the induced sputum examination, so that the patient can maintain as much understanding and comfort as possible. During the induced sputum examination, closely monitor $\mathrm{FEV}_{1}$. If the decrease of $\mathrm{FEV}_{1}$ exceeds the baseline value $20 \%$, the operation should be stopped immediately. During the operation, if the patient develops chest tightness, cough or dyspnoea, the operation should be stopped immediately and bronchodilation drugs should be given. The induced sputum should be tested as soon as possible after treatment, usually within 2 hours.

\section{Statistical analysis}

For data collection and entry, we use Epidata V.3.1 software to establish a database. All data are entered in two copies. After logical inspection, verification, cleaning and blind review, the database is locked. A statistical analysis plan is developed at the beginning of the trial design and the statistical analyst uses SPSS V.22.0 for analysis. The descriptiveness of the subjects' baseline and clinical characteristics is as follows: the measurement data use the mean and SD, and the count data use the component ratio and rate. For observation indicators, the following statistical methods are used:

Measurement data: calculate the mean and SD of each functional test index, and carry out normal inspection at the same time. For comparison between groups, independent-sample t-test was used for data conforming to normal distribution, and rank-sum test was used for non-normally distributed data. For intragroup comparison, paired t-test was used for data conforming to normal distribution, and rank-sum test was used for non-normal distribution data. When the variance is homogeneous, single-factor analysis of variance is used for comparison between multiple groups, and the Welch test is used when the variance is uneven. When $\mathrm{p}<0.05$, the difference is considered to be statistically significant.

Enumeration data: calculate the constituent ratios and rates of each index. The total effective rate is compared with the four-square table test (or exact probability method) between groups, and the Fisher's exact test $(2 \times \mathrm{C})$ is used for the comparison of the constituent ratios between groups. The rank-sum test (Mann-Whitney method) was used to compare the rank data between groups. When $\mathrm{p}<0.05$, the difference is considered to be statistically significant.

\section{DISCUSSION}

COPD is a clinical syndrome comprised of chronic bronchitis and emphysema, and the total number of patients with COPD in China is about 100 million. ${ }^{22}$ Among them, the prevalence rate of COPD in people over 40 years old is $13.7 \%$, while the prevalence rate in people over 60 years old is over $27 \%$. The number of male patients is 22 times that of female patients. COPD exacerbation is one of the most common reasons for acute hospital admissions, imposing a large burden on health resources. AECOPD is an independent risk factor for death in most patients with COPD, and its prevention and treatment level directly affect the survival of patients. ${ }^{23}$ Therefore, it is very important to optimise the diagnosis and treatment strategy of AECOPD, and the rational use of antibacterial drugs is the top priority. Patients with COPD have increased airway mucus secretion and increased airflow obstruction due to airway infection or non-infectious factors, manifested as increased dyspnoea, increased sputum volume and purulent sputum, often accompanied by increased cough and wheezing. ${ }^{24}$ When the degree of aggravation of the above symptoms exceeds the daily variation and reaches the level that requires intensive treatment or even medical treatment, it is AECOPD.

Although the reports of different researchers are quite different, more consistent results include: $60 \%$ $80 \%$ of AECOPD are caused by infection. About $50 \%$ of them can prove that the virus can be detected in its early stage; $50 \%$ of patients have bacteria detected; $5 \%-10 \%$ are infected by atypical pathogens; and $20 \%-40 \%$ of AECOPD may be caused by non-infectious factors, most of which are difficult to determine a single cause. Therefore, determining whether there is an infection and its pathogen is the basis for successful treatment. On the first to second day in the early stage of COPD exacerbation, upper respiratory tract infection-like symptoms occur first, manifested as fever, general malaise, increased cough, slightly increased sputum volume, accompanied by a certain degree of shortness of breath but rarely purulent sputum. Then the expectoration of sputum increased, the sputum gradually became thicker and the dyspnoea was obviously aggravated, accompanied by obvious wheezing.

Gut microbiota imbalance is now considered not only a potential cause of many digestive tract diseases, but also a potential cause of non-digestive tract diseases, such as obesity and neurological diseases. ${ }^{25}{ }^{26}$ The lung is actually not sterile, but contains obvious microbial communities. ${ }^{27}$ Lung flora imbalance can cause many respiratory diseases, such as COPD. ${ }^{28}$ The interaction between the intestine and lungs is called the 'gut-lung axis'. ${ }^{29} 30$ Under stress conditions such as trauma and infection, the disturbance in gut microbiota can release a large amount of active oxygen, and the intestinal barrier function will be disrupted. The bacteria enter the blood and spread through the blood to other tissues or organs of the host, and finally cause inflammation. The lung microbes are connected with intestinal bacteria. ${ }^{31}$ Gut microbiota can provide extensive protection for respiratory infections to a certain extent. Mice with reduced intestinal flora can lead to impaired immune responses and bacterial or viral infections in the respiratory tract. ${ }^{32}$ The flora can clear the lung infection of Escherichia coli through the expression of TLR $4 .{ }^{33}$ Studies have confirmed that the imbalance of intestinal flora can activate the LPS/ TLR4 pathway, thereby upregulating the lung tissue 
inflammation caused by influenza virus and aggravating the pathological state of lung tissue caused by influenza virus. ${ }^{34}$ The symbiotic bacteria in the human intestines such as bifidobacteria and lactobacilli are of great significance to the immune response of the body. Studies have found that the number of bifidobacteria and lactobacilli in the faeces of patients with COPD is significantly less than that of normal people, and the imbalance of intestinal flora is related to the incidence of acute COPD. ${ }^{1026}$ Most patients with COPD are long-term smokers, and studies have shown that chronic smoke exposure can change the structure of intestinal microbes. ${ }^{35}$ Although the biological mechanism between smoking and the intestinal environment is not clear, it is likely to be the result of comprehensive effects such as changes in the environment, host and microorganisms, such as intestinal immune damage, antigen clearance failure, acidification of gastric contents, etc. ${ }^{36}{ }^{37}$ Patients with COPD often have repeated lung infections and require long-term use of large amounts of antibiotics. Overdose of antibiotics can easily lead to imbalance of the intestinal flora. As the growth of some parasitic bacteria and yeasts in the intestine (such as Candida albicans) is inhibited, the number of bacteria (such as Proteus, Staphylococcus and Clostridium difficile) would increase. ${ }^{38}$ The use of broad-spectrum antibiotics causes collateral damage, including overgrowth of potentially pathogenic intestinal flora, which can impact the inflammatory immune responses. Patients with AECOPD frequently receive antibiotic treatment and are often subjected to mechanical ventilation, which may induce local and systemic inflammatory responses. A healthy gut microbiome produces chemical signals that can inhibit inflammation in the intestines and even the whole body, while a damaged gut microbiome may produce pro-inflammatory factors. When certain microorganisms metabolise dietary fibre, they produce large amounts of short-chain fatty acids (SCFAs). SCFAs can be transported to different places through the blood circulation to affect the recruitment and vitality of immune cells in the body (including the lungs), thereby reducing inflammation. Increased systemic inflammation is a risk factor for COPD exacerbation. Microbes also produce other messenger molecules. For example, endotoxin can stimulate immune cells to release cytokines to promote inflammation. Although the exact causes and effects are still unclear, the study of the influence of the microbiome on inflammation has been considered an important link.

Although pathogenic bacteria can be detected in the lung of a patient with AECOPD, it is difficult for some patients with AECOPD to obtain valuable lower respiratory tract secretions, and it is impossible to identify the pathogenic bacteria. Many patients have detected multiple bacteria in the sputum successively or even simultaneously, and it is difficult to determine the true pathogenic bacteria. There is a view that new strategies for COPD antibacterial treatment require attention not only to the short-term efficacy of patients, but also to the long-term prognosis of patients, including reducing the frequency of AECOPD, extending the interval between two episodes and delaying the progression of the disease. Lung dysbiosis can promote airway inflammation and reduce local immunity, and may play a role in the pathogenesis of AECOPD. Studies have shown that the microbiome profile of sputum in patients with AECOPD is related to 1-year mortality and can be used to predict prognosis. The relative abundance of Veillonococcus in the sputum of survivors is higher, while the abundance of Staphylococcus in the sputum of non-survivors is higher. ${ }^{39}$ In this study, we used metagenomic sequencing for the analysis of sputum. Metagenomic sequencing is beneficial to increase the number and proportion of pathogens read in the sequence data, and can improve the detection sensitivity of target microorganisms. The sputum microbiome may provide prognostic features, which may be used to identify patients with COPD who may benefit from close clinical monitoring after discharge following a COPD-related hospitalisation.

COPD is a chronic disease that seriously endangers human health. In recent years, with the increasingly standardised management of chronic diseases, the clinical symptoms and the number of patients with AECOPD have been effectively controlled, but the progressive decline in lung function is still not well controlled. The industry urgently needs to carry out research on new methods and new approaches for the treatment of COPD. Under the premise that the mechanism of action of pulmonary flora and intestinal flora has not been fully elucidated, this study focuses on the close relationship between intestinal flora imbalance and COPD, and reveals the relationship between intestinal microbes and lung microbes. The mechanism of action between the 'gut-lung axis' may become a new entry point for the treatment of COPD. A comprehensive understanding of the intestinal microenvironment of patients with COPD may be a promising measure for the development of effective drugs to improve the intestinal flora of patients with COPD.

\section{Trial status}

The trial was registered on 14 May 2020 and is currently in the early stage of recruitment.

\section{Ethics and dissemination}

This study has been approved by the ethics committee of Guangdong Provincial Hospital of Traditional Chinese Medicine (approval number ZF2019-219-03). We will publish and present our results at national and international conferences and in peer-reviewed journals.

\section{Author affiliations}

${ }^{1}$ School of Traditional Chinese Medicine, Jinan University, Guangzhou, China ${ }^{2}$ The Second Affiliated Hospital of Guangzhou University of Chinese Medicine, Guangdong Provincial Hospital of Traditional Chinese Medicine, Guangzhou, Guangdong, China

${ }^{3}$ Department of Respiratory Medicine, Guangdong Provincial Hospital of Traditional Chinese Medicine, Guangzhou, Guangdong, China

Contributors LD and JY wrote the first draft of the article of the study protocol, participated in the platform construction and research design, performed project 
quality control, enrolled patients and did data collection. YL and QW were responsible for writing the review and editing, developing the study protocol, and conducting and supervising the clinical study as primary investigators. YX, $\mathrm{HX}$ and $\mathrm{XC}$ contributed to the design of the study, supervised the study protocol and revised the manuscript. JY and $\mathrm{CH}$ conceived the study and enrolled patients. All authors have contributed to the design and implementation of the study. All authors read and approved the final manuscript.

Funding This work was supported by National Natural Science Foundation of China (No. 81830114, No. 81774164 and No. 82004232), Guangdong Basic and Applied Basic Research Foundation, China (No. 2020A1515010756), and Project of Administration of Traditional Chinese Medicine of Guangdong Province (No. 20201074)

\section{Competing interests None declared.}

Patient and public involvement Patients and/or the public were not involved in the design, or conduct, or reporting, or dissemination plans of this research.

Patient consent for publication Not required.

Provenance and peer review Not commissioned; externally peer reviewed.

Open access This is an open access article distributed in accordance with the Creative Commons Attribution Non Commercial (CC BY-NC 4.0) license, which permits others to distribute, remix, adapt, build upon this work non-commercially, and license their derivative works on different terms, provided the original work is properly cited, appropriate credit is given, any changes made indicated, and the use is non-commercial. See: http://creativecommons.org/licenses/by-nc/4.0/.

ORCID iD

Li Deng http://orcid.org/0000-0002-1522-4184

\section{REFERENCES}

1 Schellack N, Schellack G, Omoding R. Chronic obstructive pulmonary disease: an update. Sa Pharmaceutical Journal 2015;82:24-9.

2 Agustí A, Vogelmeier C, Faner R. COPD 2020: changes and challenges. Am J Physiol Lung Cell Mol Physiol 2020;319:L879-83.

3 Balkissoon R. Journal Club-COPD2020 Update. Global Initiative for Chronic Obstructive Lung Disease 2020 Report and the Journal of the COPD Foundation Special Edition, Moving to a New Definition for COPD: "COPDGene® 2019". J Copd F 2020;7:64-72.

4 Halpin DMG, Criner GJ, Papi A, et al. Global initiative for the diagnosis, management, and prevention of chronic obstructive lung disease. The 2020 gold science Committee report on COVID-19 and chronic obstructive pulmonary disease. Am J Respir Crit Care Med 2021;203:24-36.

5 Kherad O, Bridevaux P-O, Kaiser L, et al. Is acute exacerbation of COPD (AECOPD) related to viral infection associated with subsequent mortality or exacerbation rate? Open Respir Med J 2014;8:18-21.

6 Blasi F, Chalmers JD, Aliberti S. Copd and bronchiectasis: phenotype, endotype or co-morbidity? COPD 2014;11:603-4

7 Kirschner SK, Deutz NEP, Jonker R, et al. Intestinal function is impaired in patients with chronic obstructive pulmonary disease. Clin Nutr 2021;40:2270-7.

8 Hill SLet al. Relationship of sputum colour to bacterial load in COPD. Thorax 2000;55:A41.

9 Liu J, Ran Z, Wang F, et al. Role of pulmonary microorganisms in the development of chronic obstructive pulmonary disease. Crit Rev Microbiol 2021;47:1-12.

10 Sun Z, Zhu Q-L, Shen Y, et al. Dynamic changes of gut and lung microorganisms during chronic obstructive pulmonary disease exacerbations. Kaohsiung J Med Sci 2020;36:107-13.

11 Schroeder BO, Bäckhed F. Signals from the gut microbiota to distant organs in physiology and disease. Nat Med 2016;22:1079-89.

12 (GOLD), T.G.I.f.C.O.L.D.-s. Global strategy for diagnosis, management and prevention of COPD, 2020. Available: https:// goldcopd.org/gold-reports/

13 Paplińska-Goryca M, Nejman-Gryz P, Górska K, et al. Expression of inflammatory mediators in induced sputum: comparative study in asthma and COPD. Adv Exp Med Biol 2018;1040:101-12.

14 Guiot J, Demarche S, Henket M, et al. Methodology for sputum induction and laboratory processing. J Vis Exp 2017. doi:10.3791/56612. [Epub ahead of print: 17 Dec 2017].
15 Pan H, Guo R, Zhu J, et al. A gene Catalogue of the Sprague-Dawley rat gut metagenome. Gigascience 2018;7: giy055.

16 Jin W, Pan J, Miao Q, et al. Diagnostic accuracy of metagenomic next-generation sequencing for active tuberculosis in clinical practice at a tertiary General Hospital. Ann Transl Med 2020;8:1065.

17 Deng L, Pang P, Zheng K, et al. Forsythoside a controls influenza A virus infection and improves the prognosis by inhibiting virus replication in mice. Molecules 2016;21:524.

18 Jones PW, Harding G, Berry P, et al. Development and first validation of the COPD assessment test. Eur Respir J 2009;34:648-54.

19 Mahler DA, Wells CK. Evaluation of clinical methods for rating dyspnea. Chest 1988;93:580-6.

20 Soler-Cataluña JJ, Martínez-García MA, Román Sánchez P, et al. Severe acute exacerbations and mortality in patients with chronic obstructive pulmonary disease. Thorax 2005;60:925-31.

21 Donaldson GC, Seemungal TAR, Bhowmik A, et al. Relationship between exacerbation frequency and lung function decline in chronic obstructive pulmonary disease. Thorax 2002;57:847-52.

22 Fang X, Wang X, Bai C. Copd in China: the burden and importance of proper management. Chest 2011;139:920-9.

23 Leung JM, Chen V, Hollander Z, et al. Copd exacerbation biomarkers validated using multiple reaction monitoring mass spectrometry. PLoS One 2016;11:e0161129.

24 Zhou Y. Acute exacerbations of chronic obstructive pulmonary disease: etiological features and diagnosis. Chinese Journal of Practical Internal Medicine 2011;31:810-2.

25 Gollwitzer ES, Marsland BJ. Microbiota abnormalities in inflammatory airway diseases - Potential for therapy. Pharmacol Ther 2014;141:32-9.

26 Hu Y, Shi Q, Ying S, et al. Effects of compound Caoshi silkworm granules on stable COPD patients and their relationship with gut microbiota: a randomized controlled trial. Medicine 2020;99:e20511.

27 Willner D, Haynes MR, Furlan M, et al. Spatial distribution of microbial communities in the cystic fibrosis lung. Isme $J$ 2012;6:471-4.

28 Hussell T, Lui S, Jagger C, et al. The consequence of matrix dysfunction on lung immunity and the microbiome in COPD. Eur Respir Rev 2018;27:180032.

29 Ma Y, Yang X, Chatterjee V, et al. The Gut-Lung axis in systemic inflammation. Role of mesenteric lymph as a conduit. Am J Respir Cell Mol Biol 2021;64:19-28.

30 Willis KA, Stewart JD, Ambalavanan N. Recent advances in understanding the ecology of the lung microbiota and deciphering the gut-lung axis. Am J Physiol Lung Cell Mol Physiol 2020;319:L710-6.

31 Julian MW, Bao S, Knoell DL, et al. Intestinal epithelium is more susceptible to cytopathic injury and altered permeability than the lung epithelium in the context of acute sepsis. Int J Exp Pathol 2011;92:366-76.

32 Liang J, Sha SM, Wu KC. Role of the intestinal microbiota and fecal transplantation in inflammatory bowel diseases. J Dig Dis 2014;15:641-6.

33 Tsay T-B, Yang M-C, Chen P-H, et al. Gut flora enhance bacterial clearance in lung through Toll-like receptors 4. J Biomed Sci 2011;18:68.

34 Baharom F, Thomas S, Bieder A, et al. Protection of human myeloid dendritic cell subsets against influenza $A$ virus infection is differentially regulated upon TLR stimulation. $J$ Immunol 2015;194:4422-30.

35 Fricker M, Goggins BJ, Mateer S, et al. Chronic cigarette smoke exposure induces systemic hypoxia that drives intestinal dysfunction. JCI Insight 2018;3:e94040.

$36 \mathrm{Kim} \mathrm{M,} \mathrm{Gu} \mathrm{B,} \mathrm{Madison} \mathrm{MC,} \mathrm{et} \mathrm{al.} \mathrm{Cigarette} \mathrm{smoke} \mathrm{induces} \mathrm{intestinal}$ inflammation via a Th17 cell-neutrophil axis. Front Immunol 2019;10:75

37 Savin Z, Kivity S, Yonath $\mathrm{H}$, et al. Smoking and the intestinal microbiome. Arch Microbiol 2018;200:677-84.

38 Beauchesne M-F, Julien M, Julien L-A, et al. Antibiotics used in the ambulatory management of acute COPD exacerbations. Int $\mathrm{J}$ Chron Obstruct Pulmon Dis 2008;3:319-22.

39 Leitao Filho FS, Alotaibi NM, Ngan D, et al. Sputum microbiome is associated with 1-year mortality after chronic obstructive pulmonary disease hospitalizations. Am J Respir Crit Care Med 2019;199:1205-13.

40 Expert consensus on anti-infective therapy for acute exacerbation of chronic obstructive pulmonary disease in China. International Journal of Respiration 2019;39:1281-96. 